\title{
Autodirección y Variables Sociodemográficas y Académicas Relacionadas con la Redacción de Artículos Científicos en Estudiantes de Odontología
}

\author{
Self-Direction and Socio Demographic and Academic Factors Related \\ to Writing Scientific Papers Skills in Dental Students
}

Emilia Carrasco A. ., ; ; Eduardo Fasce H..* \& Cristhian Pérez V.

CARRASCO, E.; FASCE, E. \& PÉREZ, C. Autodirección y variables sociodemográficas y académicas relacionadas con la redacción de artículos científicos en estudiantes de odontología. Int. J. Odontostomat., 7(3):365-372, 2013.

RESUMEN: La capacidad para redactar comunicaciones científicas es considerada una competencia importante a desarrollar en estudiantes de Odontología, siendo escasa la evidencia que evalúa esta habilidad en los estudiantes de pregrado. Los objetivos fueron evaluar las habilidades para redactar comunicaciones científicas y establecer su relación con la predisposición al aprendizaje autoridirigido, factores sociodemográficos y rendimiento académico previo. Participaron 71 alumnos de segundo año de la carrera de Odontología quienes desarrollaron informes de laboratorio con un formato de publicación científica. Se diseñaron los siguientes instrumentos: pauta diseñada por los autores para evaluar la calidad de los artículos, Escala de Predisposición al Estudio Autodirigido de Fischer, King y Tague validada al español. El 15,5\% de los alumnos logró redactar una comunicación científica conforme a criterios, el $66,2 \%$ y el $91,5 \%$ de los alumnos logró desarrollar la introducción y los materiales y método, respectivamente. El 9,8\% logró exponer los resultados en relación a los objetivos propuestos y sólo el $7 \%$ de los alumnos planteó conclusiones pertinentes. Los hombres $(M=5,23 ; D E=0,54)$ mostraron mejor desempeño que las mujeres $(M=4,93 ; D E=0,67), p<0,03$, y no hubo relación con tipo de establecimiento de origen, notas de enseñanza media, prueba de selección universitaria y predisposición al aprendizaje independiente. Se comprueba la carencia de habilidades para redactar artículos científicos en estudiantes de Odontología, generando la necesidad de propiciar acciones remediales.

PALABRAS CLAVE: aprendizaje auto-dirigido, habilidades científicas, estudiantes odontología.

\section{INTRODUCCIÓN}

Dentro de las competencias genéricas que las carreras de Odontología aspiran a desarrollar en sus estudiantes, está la capacidad de realizar investigación. Competencia de difícil desarrollo en las mallas curriculares en pregrado, por cuanto, el desarrollo de actividades de investigación dentro de la carrera requiere de docentes formados en investigación básica y/o clínica con líneas de investigación activas, condición de escaso desarrollo en las universidades chilenas. Es por esto que la utilización de instancias formativas convencionales para la aplicación del mé- todo científico, la observación, el análisis de fenómenos biológicos que se pueden aplicar a la clínica, el apropiado manejo de datos obtenidos en el laboratorio, la comunicación de los hallazgos, y con el objetivo fundamental de fomentar la actitud creativa y crítica por parte del estudiante de Odontología como contraparte a la simple repetición de información, siguiendo caminos preestablecidos, inamovibles e incuestionables, constituyen instancias ideales que se deben aprovechar en los laboratorio en las asignaturas de ciencias básicas, para el desarrollo de competencias

\footnotetext{
* Departamento Educación Medica, Universidad de Concepción, Concepción, Chile.

${ }^{* *}$ Facultad de Odontología, Universidad San Sebastían, Concepción, Chile.

Investigación financiada por proyecto Fondecyt 1110718.
} 
en investigación científica. En la literatura científica, es posible encontrar numerosas publicaciones realizadas en el extranjero en áreas de ingenieria y ciencias básicas, principalmente en química, referentes a la introducción de nuevos aspectos en los laboratorios que van desde el incentivo de actitudes críticas, de aplicación de conocimientos a la vida real, hasta la incorporación de actividades que preparen al alumno en la realización de actividades científicas. Se observa una mayor motivación de los estudiantes frente al desarrollo de los laboratorios (Pringle \& Henderleiter, 1999), adquisición de experiencia en investigación (Kharas, 1997) permite el uso del pensamiento creativo en la resolución de problemas (Silverstein et al., 1997) permite elevar el aprendizaje de las destrezas básicas del laboratorio químico (González et al., 2002) y aprendieron en forma apropiada a plantear un proyecto, consultar bibliografía disponible, aplicar conocimientos aprendidos en la clase, interpretar y analizar los datos del laboratorio, comunicar los resultados del proyecto y trabajar en grupo (Rodríguez-Sandoval et al., 2010). Sin embargo, en las ciencias del área de la salud en general, y en Odontología en particular, no existen antecedentes que den cuenta del impacto en los alumnos, frente a incentivos en dirección al desarrollo de actividades investigativas en el programa regular de la carrera.

Por lo tanto, se propone con este estudio, evaluar las habilidades de comunicación escrita con formato de publicación científica y la relación de estas, con la capacidad de aprendizaje auto-dirigido y características socio-demográficas de los estudiantes de segundo año de Odontología.

\section{MATERIAL Y METODO}

La muestra, elegida a través de muestreo no probabilístico por accesibilidad, quedó constituida por los 71 estudiantes que cumplieron con los criterios de inclusión, de los cuales $47(66,20 \%)$ eran mujeres y $24(33,80 \%)$ eran hombres, con edades entre los 20 y los 33 años $(M=22,06 ; D E=2,46)$. De éstos, 13 $(18,31 \%)$ provenían de establecimientos municipalizados, $29(40,85 \%)$ de establecimientos particulares subvencionados y $24(33,80 \%)$ de establecimientos particulares pagados, con cinco casos que no aportaron esta información. Los criterios de inclusión utilizados fueron: ser alumno regular de la asignatura de Infectología Oral Facultad de Odontología, Universidad San Sebastían, haber completado el semestre académico, cumpliendo con los requisitos mínimos de asistencia de $80 \%$ de las actividades programadas para el semestre, haber entregado los informes de laboratorio y haber firmado en consentimiento informado diseñado para este estudio. Los criterios de exclusión fueron: no estar inscrito en la asignatura de Infectología Oral, haber suspendido o congelado el semestre académico, no haber cumplido con la asistencia a los laboratorios, no haber entregado el informe de laboratorio y no haber firmado el consentimiento informado.

Para la evaluación de los informes de laboratorio se elaboró y aplicó una pauta cuyos ítemes fueron categorizados a través de rúbricas. Esta pauta está compuesta por seis ítemes que presentan los atributos o aspectos propios de una comunicación científica escrita, vale decir, resumen, introducción, objetivos, materiales y método y conclusiones. El sexto ítem contempla la evaluación del trabajo independiente del alumno, según las características que presenta el informe. Se evalúa en este ítem: si fue capaz de realizar un análisis de publicaciones científicas, realizar un análisis crítico de la información, y si propone aplicaciones clínicas del experimento realizado en el laboratorio. Cada uno de estos ítems presenta tres alternativas de puntuación ( $1=$ deficiente; $2=$ bueno y $3=$ sobresaliente). Cada ítem que contempla la pauta tiene una ponderación distinta para la obtención del puntaje, aplicándose una escala con exigencia del $50 \%$ para la obtención de la nota. Las ponderaciones de los ítems son los siguientes; introducción, objetivos y material y métodos $10 \%$, los resultados $20 \%$, las conclusiones $30 \%$ y el ítem trabajo independiente $20 \%$.

Se aplicó la escala de predisposición al aprendizaje independiente desarrollada por Fischer, traducida, validada y determinada su confiabilidad en estudiantes de medicina (Fasce et al., 2011). Esta escala está compuesta por 40 ítems que presentan atributos, habilidades o aspectos motivacionales que son característicos de los aprendices autónomos. El participante debe indicar el grado en que el ítem representa una característica de él, utilizando una de cinco alternativas (1=muy en desacuerdo; $2=$ en desacuerdo; $3=$ indeciso; 4=de acuerdo y 5= muy de acuerdo). La Escala de Aprendizaje Autodirigido (Escala General) presenta una estructura de cinco factores o subescalas que tributan al aprendizaje autodirigido: una dimensión procedimental ("Planificación del Aprendizaje"), aspectos actitudinales ("Deseo por Aprender" y "Autoconfianza"), y aspectos cognitivos vinculados a la autonomía ("Autogestión” y “Autoevaluación”). 
Para el procesamiento estadísticos de los datos, éstos primero fueron ingresados a una planilla Excel y luego fueron importados y analizados en el paquete estádistico STATA S.E. 11.0.

Como primer paso se realizó un análisis descriptivo de las variables sociodemográficas para describir la muestra, identificando las frecuencias absolutas y relativas de la variable sexo y tipo de colegio, en tanto variables categóricas, y la media aritmética, desviación estándar, mínimo y máximo de la variable edad, en tanto variable numérica.

Posteriormente, se realizó un análisis - también descriptivo - del desempeño de los alumnos identificando tanto la tendencia central (media aritmética) y dispersión de los mismos (desviación estándar, mínimo y máximo), como su distribución de frecuencias según tramo de rendimiento en general y por área de desempeño.

Además, se realizó un análisis de las medidas de tendencia central y dispersión de los antecedentes académicos de los estudiantes (NEM y PSU) y de los puntajes de la Escala de Aprendizaje Autodirigido, a la que previamente se evaluó su consistencia interna mediante el coeficiente Alfa de Cronbach.

Finalmente, en cuanto a análisis bivariados, se empleó $t$ de Student, para muestras independientes en base a un contraste unilateral, para identificar diferencias en el desempeño en la elaboración del paper según sexo, y ANOVA de un factor para identificar diferencias según establecimiento. También se ocupó el coeficiente $r$ de Pearson para relacionar este desempeño con los antecedentes académicos y el aprendizaje autodirigido.

\section{RESULTADOS}

La variable dependiente del estudio, el desempeño de los alumnos en el informe de investigación, se operacionalizó a través de notas en escala de 1 a 7 , las cuales oscilaron entre el 3,5 y el 6,2 , con un promedio de 5,03 y una desviación estándar de 0,64. En la Figura 1. se observa que las notas se distribuyeron mostrando una moderada asimetría negativa. El $66 \%$ de los alumnos obtuvo nota igual o superior a 5 , de los cuales sólo el 15,5\% alcanzó una calificación superior a 5,7, vale decir, lograron redactar una publicación científica según la pauta utilizada.

La principal debilidad observada en este grupo de alumnos que obtuvieron calificaciones superiores o iguales a 5,7, fue no haber logrado redactar el resumen del informe en conformidad a la pauta de evaluación. Este mismo grupo de alumnos, logró un mejor cometido en la formulación de objetivos, en la redacción de resultados y en las conclusiones.

En general, la mayoría de los 71 estudiantes de la muestra redactaron una introducción según los atributos que están contemplados en la pauta, así como también, la exposición de los materiales y métodos del experimento, cumpliendo con la descripción detallada de los materiales y procedimientos realizados y que correspondieron al $66,2 \%$ y el $91,5 \%$ de los alumnos con puntuación 2 y 3 , respectivamente (ver Tabla I). Sin embargo, en la redacción de los resultados y las conclusiones, la puntuación máxima fue logrado por menos del $10 \%$ de los alumnos; $9,8 \%$ en el caso de la redacción de resultados y $7 \%$ en relación a las conclusiones (Tabla I).

Otro aspecto deficitario fue la carencia de consultas bibliograficas en relación al tema tratado: sólo 5 estudiantes se refirieron a la bibliografía científica disponible (ver Tabla II).

En los ítemes destinados a evaluar el nivel de estudio independiente los resultados fueron insatisfactorios: sólo 3 alumnos, que corresponden al $4,2 \%$ de la muestra, esbozaron un análisis crítico de la información confrontándola con los resultados obtenidos y presentaron una aplicación clínica de la información obtenida del experimento realizado en el laboratorio (Tabla II)

Tabla I. Distribución porcentual de los puntajes obtenidos en las diferentes secciones del reporte de investigación.

\begin{tabular}{lccc}
\hline Sección & Puntuación 1 & Puntuación 2 & Puntuación 3 \\
\hline Introducción & 33,8 & 59,15 & 7 \\
Objetivos & 8,45 & 66,19 & 25,35 \\
Materiales y Método & 8,45 & 15,49 & 76 \\
Resultados & 63,38 & 26,76 & 9,8 \\
Conclusiones & 77,46 & 15,49 & 7 \\
\hline
\end{tabular}


Tabla II. Relación de los aspectos relativos al trabajo independiente demostrado en el informe y el puntaje obtenido por los alumnos según la pauta.

\begin{tabular}{lccc}
\hline Aspecto/ Puntuación & Puntuación 1 & Puntuación 2 & Puntuación 3 \\
\hline Trabajo Independiente & $54(76,05)^{*}$ & $12(16,9)$ & $5(7)$ \\
Análisis Publicaciones científicas & $60(84,5)$ & $9(12,67)$ & $5(7)$ \\
Análisis crítica de la información & $63(88,7)$ & $5(7,04)$ & $3(4,2)$ \\
Aplicabilidad del experimento & $56(78,87)$ & $12(16,9)$ & $3(4,2)$ \\
\hline
\end{tabular}

*número y porcentaje de alumnos.

En cuanto a las variables independientes referidas al desempeño académico previo del estudiante, incluyeron el puntaje en la Prueba de Selección Universitaria (PSU), PSU de Matemática (PSUM), PSU de Lenguaje y Comunicación (PSUL), PSU de Ciencias (PSUC), y el promedio de Notas de Enseñanza Media (NEM). Los estadísticos descriptivos de éstas pruebas se presentan en la Tabla III.

En cuanto a la autonomía en el aprendizaje, evaluado a través de la Escala de Aprendizaje Autodirigido, los estadísticos descriptivos de su escala general y de sus cinco subescalas se describen a continuación (Tabla IV). Asimismo, en esta Tabla se exponen las confiabilidades de éstas, todas las cuales fueron adecuadas.

\section{Análisis bivariados}

Para evaluar la relación del rendimiento obtenido en el informe con las variables dependien- tes académicas, sociodemográficas y el aprendizaje autodirigido, se decidió realizar un análisis bivariado, utilizando pruebas estadísticas paramétricas, ya que el tamaño muestral permite el uso de éstas pruebas independiente de la distribución muestral (Pardo et al., 2009). Por otro lado, como se observó antes, la asimetría de la variable dependiente - la calificación en el informe - tampoco era pronunciada (Fig. 1), por lo que el uso de estas pruebas es pertinente.

En primer lugar, para evaluar la relación del desempeño de los alumnos con el sexo, se comparó el desempeño de hombres y mujeres utilizando la prueba t de Student para muestras independientes en base a un contraste bilateral. Al hacerlo, se encontró que existían diferencias estadísticamente significativas entre hombres (M $=5,23 ; \mathrm{DE}=0,54)$ y mujeres $(\mathrm{M}=4,93 ; \mathrm{DE}=0,67)$, $\mathrm{t}(69)=1,93 ; p<0,03$, obteniendo los primeros un mejor desempeño.

Tabla III. Descriptivos de los antecedentes académicos previos de los estudiantes de odontología.

\begin{tabular}{lccccc}
\hline & $\boldsymbol{n}$ & $\boldsymbol{M}$ & $\boldsymbol{D E}$ & Mín. & Máx. \\
\cline { 2 - 6 } PSU Gral. & 70 & 629,84 & 41,22 & 537,50 & 769,00 \\
PSUM & 70 & 633,69 & 44,35 & 536,00 & 769,00 \\
PSUL & 70 & 627,24 & 58,58 & 498,00 & 801,00 \\
PSUC & 66 & 625,45 & 39,86 & 552,00 & 734,00 \\
NEM & 66 & 6,15 & 0,36 & 5,30 & 6,90 \\
\hline
\end{tabular}

Tabla IV. Estadísticos descriptivos de la Escala de Predisposición al Aprendizaje Independiente.

\begin{tabular}{lccccc}
\hline & & $\boldsymbol{M}$ & $\boldsymbol{D E}$ & Mín. & Máx. \\
\cline { 2 - 6 } Escala General & 0,90 & 153,17 & 14,36 & 115 & 183 \\
Planificación del aprendizaje & 0,83 & 36,08 & 6,30 & 19 & 49 \\
Deseo de aprender & 0,82 & 24,24 & 3,54 & 15 & 30 \\
Autoconfianza & 0,76 & 38,62 & 3,77 & 29 & 45 \\
Autogestión & 0,65 & 38,42 & 3,34 & 30 & 45 \\
Autoevaluación & 0,56 & 15,80 & 2,35 & 10 & 20 \\
\hline
\end{tabular}



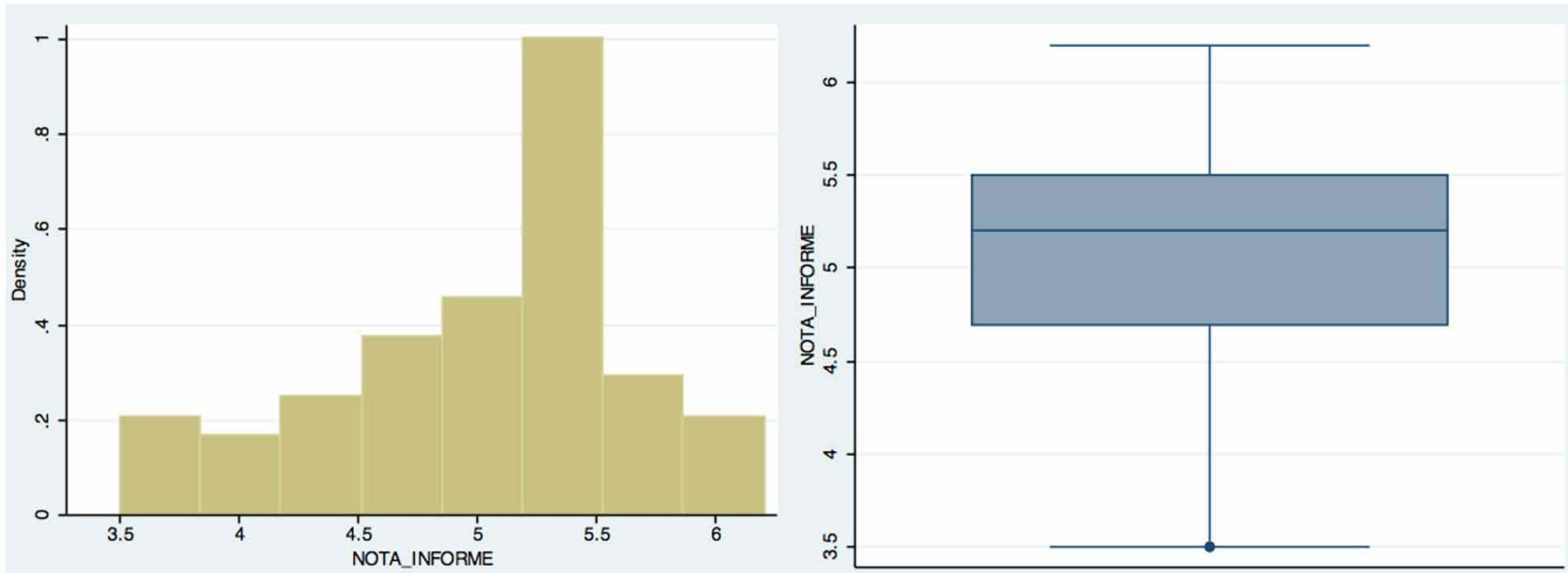

Fig. 1. Distribución de las calificaciones obtenidas en el informe por los alumnos de odontología.

En segundo lugar, se compararon los desempeños de los alumnos según el tipo de colegio del que habían egresado, para lo cual se usó la prueba ANOVA de un factor, encontrándose que no había diferencias estadísticamente significativas entre las calificaciones obtenidas por los egresados de establecimientos municipalizados $(M=4,92$; $D E=0,75)$, particulares subvencionados $(M=4,98 ; D E=0,66)$ y particulares pagados $(M=5,18 ; D E=0,60), F(2,63)$ $=0,95 ; p=0,39$.
En cuando a la relación del desempeño en el informe con los antecedentes académicos previos, ésta se evaluó a través del coeficiente de correlación de Pearson, en base a un contraste unilateral. Sin embargo, los resultados mostraron que no había relación ni con la NEM ni con los puntajes PSU (Tabla V).

Por último, se evaluó la relación de esta calificación con los niveles de aprendizaje autodirigido de los alumnos, sin encontrarse relaciones estadísticamente significativas (Tabla VI).

Tabla V. Correlación entre la calificación en el informe y los antecedentes académicos de los alumnos de odontología.

\begin{tabular}{lcccccc}
\hline & $\mathbf{1}$ & $\mathbf{2}$ & $\mathbf{3}$ & $\mathbf{4}$ & $\mathbf{5}$ & $\mathbf{6}$ \\
\cline { 2 - 7 } Nota del informe & - & & & & & \\
NEM & 0,06 & - & & & & \\
PSU Gral. & $-0,09$ & $0,34^{* *}$ & - & & \\
PSUL & $-0,07$ & $0,27^{*}$ & $0,88^{* * *}$ & - & - & - \\
PSUM & $-0,04$ & 0,23 & $0,73^{* * *}$ & $0,34^{* *}$ & - \\
PSUC & $-0,04$ & $0,35^{* *}$ & $0,55^{* * *}$ & $0,36^{* *}$ & $0,61^{* * *}$ & - \\
\hline${ }^{*} \mathrm{p}<0,05 ;{ }^{* *} \mathrm{p}<0,01 ;{ }^{* * *} \mathrm{p}<0,001$. & & & &
\end{tabular}

Tabla VI. Correlación entre la calificación en el informe y los niveles de aprendizaje autodirigido de los alumnos de odontología.

\begin{tabular}{lcccccc}
\hline & $\mathbf{1}$ & $\mathbf{2}$ & $\mathbf{3}$ & $\mathbf{4}$ & $\mathbf{5}$ & $\mathbf{6}$ \\
\cline { 2 - 7 } Nota del informe & - & & & & & $\mathbf{7}$ \\
Escala General & 0,07 & - & & & & \\
Planificación del aprendizaje & 0,18 & $0,83^{* * *}$ & - & & & \\
Deseo de aprender & $>-0,01$ & $0,72^{* * *}$ & $0,46^{* * *}$ & - & & - \\
Autoconfianza & $-0,01$ & $0,76^{* * *}$ & $0,56^{* * *}$ & $0,38^{* *}$ & - & $0,46^{* * *}$ \\
Autogestión & 0,03 & $0,71^{* * *}$ & $0,40^{* * *}$ & $0,42^{* * *}$ & $0,47^{* * *}$ & - \\
Autoevaluación & $-0,04$ & $0,57^{* * *}$ & $0,24^{*}$ & $0,42^{* * *}$ & $0,35^{* *}$ & 0 \\
\hline
\end{tabular}

${ }^{*} p<0,05 ;{ }^{* *} p<0,01 ;{ }^{* * *} p<0,001$. 


\section{DISCUSIÓN}

El resultado más significativo de este estudio es que la mayor parte de los estudiantes, demostraron carecer de las habilidades para redactar con propiedad todas las secciones que demanda una comunicación científica. Estos resultados fueron indepedientes del rendimiento académico exhibido en sus estudios preuniversitarios, vale decir, PSU y notas de enseñanza media. Tampoco tuvieron relación con el tipo de establecimiento educacional del cual provenían ni de la predisposición al aprendizaje autodirigido. Sólo se relacionó el desarrollo de la habilidad para redactar comunicaciones científicas con el género, alcanzando mejores resultados los hombres sobre las mujeres.

El mejor aprovechamiento de las actividades de laboratorio en las ciencias básicas impartidas en los primeros años de las carreras del área de la salud, para promover una mejor formación en habilidades en investigación, requiere de la reformulación de los mismos, en términos que los estudiantes desarrollen proyectos de investigación, de tal modo que en el laboratorio sea aplicado el método científico. Siendo la comunicación de los resultados, un aspecto fundamental para la adquisición de habilidades de escritura científica por parte del alumno, lo cual enriquece su formación en ciencias, se impone introducir nuevas estrategias que reviertan las carencias demostradas con el método tradicional basado en informes de laboratorio.

González et al., reportan los beneficios obtenidos en los estudiantes de química, al reemplazar los trabajos prácticos convencionales por una investigación dirigida, donde la fase final del trabajo de laboratorio corresponde a la fase comunicativa, en la cual el estudiante debe analizar los resultados y elaborar un informe con conclusiones y recomendaciones. Esta es una fase de suma importancia, porque es la instancia para que el alumno analice los resultados obtenidos, reflexione sobre el trabajo realizado, desarrolle generalizaciones y ejercite sus habilidades comunicativas. Ellos observaron que los alumnos del grupo que realizaron un trabajo investigativo obtuvieron mejores calificaciones en la ejecución de las destrezas experimentales y en el trabajo independiente, comparado con el grupo de alumnos que desarrollaron los laboratorios en forma convencional.

Por el contrario, en nuestro estudio no se observó relación entre la calificación obtenida y los atri- butos de trabajo independiente en este grupo de alumnos de Odontología. Fue posible observar que los estudiantes escasamente revisaron la bibliografía disponible referente al tema tratado en cada laboratorio y tampoco leyeron artículos científicos en el área de odontología o microbiología, cuestión fundamental para entender la dinámica de una publicación científica. Esto debido, en primer término, a que un alto porcentaje de los alumnos no desarrolló el informe con los atributos deseables en las secciones de resultados y conclusiones de la investigación. En segundo término, debido a la carencia de aportes bibliográficos al desarrollo de la introducción de los informes y, finalmente, por no haber desarrollado la capacidad de autoprendizaje.

Estos resultados coinciden con los reportados por Robinson et al. (2007) quienes comunican la dificultad que implica para los estudiantes de pregrado en facultades de medicina en el Reino Unido, redactar proyectos de investigación, los cuales son considerados por los estudiantes como extensos y burocráticos e implican destinar demasiado tiempo y esfuerzo.

En la literatura existen antecedentes relativos a los cambios que se producen en los estudiantes al incorporar un modelo basado en investigación, tanto en los laboratorios, como en el desarrollo de una asignatura completa; el estudiante aumenta su pensamiento independiente, su capacidad de análisis critico, su interés por la materia, su capacidad de retención de la información a largo plazo, entre otros aspectos (Newton et al., 2006). Por el contrario, en el presente estudio, los estudiantes de segundo año de odontología, no logran desarrollar, en un semestre habilidades necesarias de autoaprendizaje para escribir correctamente una publicación científica, por lo tanto, se puede inferir que, no lograron adquirir habilidades de pensamiento crítico frente a la información disponible y obtener conclusiones frente a los datos emanados de la experiencia en el laboratorio de microbiología oral.

Por otro lado, en este estudio, los alumnos que desarrollaron habilidades en escritura científica obteniendo una calificación igual o superior a 5,7, y que corresponden al $15,49 \%$ del total, fueron predominantemente del sexo masculino (54,54\%). Las calificaciones más altas fueron obtenidas por los hombres de la muestra; hombres $(M=5,23 ; D E=0,54)$ y mujeres ( $M$ $=4,93 ; \mathrm{DE}=0,67), p<0,03$. En la literatura científica disponible no se reportan diferencias de género en el rendimiento de los alumnos, al introducir una activi- 
dad de investigación científica en un laboratorio. Sin embargo, los estudios publicados en este ámbito, provienen de áreas en que los hombres son más numerosos en los cursos como es el caso de ingenierias, química, entre otros. En este contexto, otras diferencias de género se han descrito en la percepción de los alumnos a la introducción en los laboratorios de química de actividades basados en casos de estudio (Pringle \& Henderleiter), lo que modifica favorablemente la actitud hacia el laboratorio y por lo tanto, se traduce en el mayor aprendizaje del estudiante en las ciencias básicas. Las mujeres de la muestra estudiada manifestaron los beneficios de la actividad pero significativamente más que los hombres y evidenciaron los defectos e inconvenientes de las nuevas actividades de laboratorio introducidas, verbalizando las expectativas insatisfechas en el laboratorio. Estas diferencias de género observadas por los autores fueron resultados no esperados por ellos, concluyendo en ese estudio la necesidad de indagar más en el tema.

Las otras variables medidas, vale decir, edad, notas de enseñanza media, puntajes obtenidos en la PSU y establecimiento educacional del cual provenían, no presentaron relaciones estadisticamente significativas con la calificación obtenida en el informe.

\section{CONCLUSIONES}

La evaluación de la estrategia instruccional de desarrollo de informes de laboratorio con un formato de comunicación científica, realizada en este estudio, respondió a la iniciativa de los autores por estimular a los alumnos de segundo año de la carrera de Odontología, en el estudio independiente para la adquisición de habilidades de redacción científica. Los resultados muestran que los alumnos estudiados en general, por sí solos no desarrollan la capacidad para realizar una comunicación científica por escrito situación muy distinta de lo que sucede en estudiantes de otras áreas, como se muestra en la literatura disponible, probablemente debido al curriculum de Odontología caracterizado por un menor énfasis en la investigación científica y más énfasis en información práctica.

Los resultados de este estudio muestran que los alumnos del sexo masculino tendrían mayores habilidades para redactar una comunicación científica con los atributos contemplados en la pauta diseñada por los autores, por lo tanto, por consiguiente tendrían mayores habilidades de trabajo independiente.en esta muestra que las alumnas.

CARRASCO, E.; FASCE, E. \& PÉREZ, C. Self-direction and socio demographic and academic factors related to writing scientific papers skills in dental students. Int. J. Odontostomat., 7(3):365-372, 2013.

ABSTRACT: In the educational development of dental students, the ability to write scientific papers is considered an important skill. Evidence that assesses this skill in undergraduates is scarce. The aims were to evaluate scientific communication writing skills and establish their relationship of predisposition to self-directed learning, to socio demographic factors and to prior academic performance. Seventy-one students in their second year of dentistry developed laboratory reports in a scientific publication format. Instruments: pattern designed by the authors to evaluate the quality of the articles, Predisposition Scale Self-Directed Study Fischer, King and Tague validated in Spanish. In 15.5\% of students achieved scientific writing in accordance with criteria. $66.2 \%$ and $91.5 \%$ of students achieved the introduction and development of materials and methods, respectively. In $9.8 \%$ were able to present the results achieved in relation to objectives, and only $7 \%$ of students presented relevant conclusions. Men $(M=5.23, S D=0.54)$ showed better performance than women $(\mathrm{W}=4.93$, $\mathrm{SD}=0.67), \mathrm{p}<0.03$, and there was no connection with the educational establishment, high school grades, college admission selection exam, and independent learning preparedness. The lack of ability to write scientific papers in dental students at the Universidad San Sebastián, generates the need to provide remedial actions.

KEY WORDS: self directed learning, scientific skills, dental students.

\section{REFERENCIAS BIBLIOGRÁFICAS}

Fasce, H. E.; Pérez, V. C.; Ortiz, M. L.; Parra, P. P. \& Matus, B. O. Estructura factorial y confiabilidad de la escala de aprendizaje autodirigido de Fisher, King \& Tague en alumnos de medicina chilenos. Rev. Med. Chile, 139(11):142834, 2011.
González, H.; Spengler, I. \& Vidal, G. Aprendizaje activo de destrezas básicas del laboratorio químico. J. Science Edu., 3(1):34-6, 2002.

Kharas, G. A New Investigative Sophomore Organic 
Laboratory Involving Individual Research Projects. J. Chem. Educ., 74(7):829-31, 1997.

Newton, T.; Tracy, H. \& Prudenté, C. A Research-Based Laboratory Course in Organic Chemistry. J. Chem. Educ., 83(12):1844-9. 2006.

Pardo, A.; Ruiz, M. A. \& San Martín, R. Análisis de datos en ciencias sociales y de la salud I. Madrid, Síntesis, 2009.

Pringle, D. \& Henderleiter, J. Effects of Context-Based Laboratory Experiments on Attitudes of Analytical Chemistry Students. J. Chem. Educ., 76(1):100-6, 1999.

Robinson, L.; Drewery, S.; Ellershaw, J.; Smith, J.; Whittle, S. \& Murdoch-Eaton, D. Research governance: Impeding both research and teaching? A survey of impact on undergraduate research opportunities. Med. Educ., 41(8):729-36, 2007.

Rodriguez-Sandoval, E.; Vargas-Solano, E. M. \& Luna-Cortés, J. Evaluación de la estrategia "aprendizaje basado en proyectos. Educ. Educ., 13(1):13-25, 2010.

Silverstein, T. P.; Hudak, N. J.; Chapple, F. H.; Goodney, D. E.; Brink, C. P. \& Whitehead, J. P. Scientific Communication and the Unified Laboratory Sequence. J. Chem. Educ., 74(2):150-2, 1997.
Dirección para Correspondencia:

Dra. Emilia Carrasco A.

Facultad de Odontología

Universidad San Sebastián

Lientur 1457

Concepción

CHILE

Email: ecarrasc@udec.cl

Recibido : 10-06-2013

Aceptado: 10-08-2013 